УДК 004.02, 621.039

МЕТОДИКА ПОИСКА ОПТИМАЛЬНОЙ КОНФИГУРАЦИИ СИСТЕМЫ АТОМНЫХ СТАНЦИЙ С УЧЕТОМ ВЛИЯНИЯ РИСКОВ, КАПИТАЛЬНЫХ ЗАТРАТ И УЩЕРБОВ ОТ АВАРИЙ

Расторгуев Иван Александрович

к.ф.-м.н., старший научный сотрудник, e-mail: rastorguev_ia@nrcki.ru

Щепетина Татьяна Дмитриевна,

к.т.н., нач. лаборатории «Перспективных концепций», e-mail: $\underline{\text { Schepetina_TD@nrcki.ru }}$

НИЦ «Курчатовский институт»

123182, Москва, пл. Курчатова, д.1,

\begin{abstract}
Аннотация. Задача поиска оптимального спектра мощностей атомных реакторов для развивающейся энергосистемы страны с большой долей вклада АС формализована и представлена в виде задачи многокритериальной оптимизации. Оценивается снижение потенциального ущерба от различных факторов риска и затрат на строительство при максимальной выработке электроэнергии. Предложена концепция поиска решения при помощи генетического алгоритма. На основе проведенных расчетов даны рекомендации по оптимизации мощностного ряда реакторных установок (РУ), которые позволят снизить совокупные риски эксплуатации АС при стратегическом планировании.
\end{abstract}

Ключевые слова: атомная энергетика, реакторные установки, единичная мощность блока, безопасность, ущерб, риск, капитальные вложения, минимизация ущерба, эволюционный алгоритм, оптимизация.

Цитирование: Расторгуев И.А., Щепетина Т.Д Методика поиска оптимальной конфигурации системы атомных станций с учетом влияния рисков, капитальных затрат и ущербов от аварий // Информационные и математические технологии в науке и управлении. 2021. № 2 (22). С. 77-88. DOI:10.38028/ESI.2021.22.2.008

Введение. Безопасность атомной энергетики как Системы становится трансграничной проблемой всего мирового сообщества. Крупные аварии разного рода стали реальной опасностью существования человечества. Побудительными мотивами для написания статьи стали необходимость анализа тенденций развития атомной энергетики и предполагаемых путей реализации, сопряженных с управленческими решениями и недостаточно системным подходом к учету интегрального риска по жизненному циклу атомных станций (AC).

Очевидно, что стратегии развития могут составляться с разными концептуальными установками, а также с постановкой реальных или ошибочных приоритетов. Задача предложенного материала - с помощью расчетных моделей показать, как учет или неучет различных системных рисков проектов $\mathrm{AC}$, а особенно риска возможного аварийного ущерба при планировании приводят к существенно различным структурным составам АС будущей Системы атомной энергетики и, соответственно, к разным уровням угроз в будущем.

Часто проектировщики строящихся АC «стараются не замечать» возрастающей технической аварийности, тогда как их главное назначение - видеть всю картину целиком. С учетом множества рисков, окружающих перспективные проекты АС и сопровождающих их по всему жизненному циклу, стратегический выбор состава АС для обеспечения стабильного и безопасного функционирования атомного (и не только) энергокомплекса является многопараметрической и даже междисциплинарной задачей оптимизации. Как правило, есть несколько ключевых параметров/«требований» по экономичности, надежности и безопасности, которые относятся как к финансовым категориям, так и нефинансовым, т.е. «немонетизируемым». 
Есть два основных направления/«возможности» развития атомного реакторостроения сооружение АС с блоками большой мощности (блоки по 700 - 2000 МВт эл.) или АС с РУ малой и средней мощности (соответственно в интервалах $10-100$ и $300-700$ МВТ эл). Каждое «требование» и «возможность» имеют свои плюсы и минусы, найти грамотный баланс которых необходимо при стратегическом планировании и определенном целеполагании.

Задача выбора компоновки проектируемой АС осложняется разнонаправленностью требований и средств их удовлетворения: например, стремление построить дешевую АС неизбежно приведет к экономии на системах безопасности. Стремление создать совершенно безопасную АC приводит к её высокой стоимости. Далее вступают в игру показатели стабильности энергоснабжения (необходимый резерв энергосистемы и её устойчивость к внезапным отключениям энергогенерации большой мощности); возможности по приему большой мощности и возможности площадки размещения по отводу сбросного тепла; показатели совокупного риска, квалификация персонала и приемлемость обществом [1] *), и многие другие показатели. (*)B английском языке это иногда называют NIMBY - not in my back yard - «Не у меня на заднем дворе».)

1. Основные определения. В данном исследовании применен дифференцированный подход к оценке вероятного ущерба от крупной аварии для блоков АС различной мощности в соответствии с «принципом Хаттори» [2], гласящем, что при снижении мощности блока в 10 раз его интегральная безопасность повышается в 1000 раз. Рассмотрены блоки АС условной мощности 1000, 500, 100 и 10 МВт эл.

Обычно под термином «риск» в современном определении понимается либо вероятность неблагоприятного события, либо возможные серьезные последствия такого события или аварии объекта, либо их произведение. Это понятие можно применить тогда, когда вероятность инцидента с большей или меньшей точностью может быть определена и ущерб квантифицирован (оцифрован). Но если рассматриваются комплексные негативные последствия, то квантификации поддается лишь малая их часть и её денежное выражение не будет воспринято как истинное. Что касается вероятности, то не считаются вероятными довольно многие события вплоть до их наступления.

В данном исследовании все категории риска учтены как «возможность неблагоприятного исхода по определенному параметру» и представлены как проценты/баллы, выставленные экспертами при анкетном опросе. Затем значения с наибольшей повторяемостью были введены в качестве исходных данных в оптимизационную программу, описание которой представлено ниже.

2. Подход к оптимизации. Задача поиска оптимального набора блоков РУ для выбранного размера Системы АЭ (или как более частный случай - малого государства, изолированной энергосистемы или площадки строительства $\mathrm{AC}$ ) формализована и представлена в виде задачи многокритериальной оптимизации: снижения потенциального ущерба от различных факторов риска, затрат на строительство при максимальной выработке электроэнергии. Решение задачи было найдено численно, при помощи эволюционного алгоритма.

Известно, что любые сложные задачи, в том числе касающиеся масштабной атомной энергетики, решаются, как правило, в условиях значительных неопределенностей и для этого необходимо применение современных вычислительных технологий.

Существуют различные алгоритмы поиска решения с помощью оптимизационных процедур, например, представленные в Интернете коды PEST [3] и Ostrich [4]. Из-за их долговременных расчётов было решено разработать собственные коды для оптимизации на языке $\mathrm{C}++$ с использованием генетического алгоритма. 
Генетические алгоритмы (ГА) [5, 8 - 10] представляют собой адаптивные методы поиска, при которых реализуются эволюционные вычисления, имеющие основой генетические процессы биологических организмов. Они являются одними из самых современных приемов решения многокритериальных оптимизационных задач (МКО). Это эвристический алгоритм поиска, используемый для решения задач оптимизации и моделирования путем последовательного подбора, комбинирования и вариации искомых параметров с помощью механизмов, напоминающих биологическую эволюцию. Отличительной особенностью генетического алгоритма является способ поиска новых точек путем комбинирования и «мутации» наилучших решений-кандидатов. В ГА используются генетические операторы (такие как скрещивание и мутации), реализующие переборную часть ГА, и эволюционный оператор отбора. Смысл параметров зависит от характера решаемой конкретной задачи. Его применение к задачам, связанным с АЭС, было продемонстрировано в [1].

Описание условий применения эволюционного алгоритма для задач МКО при подборе оптимального набора РУ

Основная цель данной задачи - выявить оптимальный по рангу мощности набор реакторных установок (РУ) при следующих условиях. В качестве поискового набора предлагаются 4 ранга РУ:

$$
\begin{aligned}
& \text { мощностью } 1000 \text { МВт }\left(n_{1}\right) \text { - тип №1; } \\
& \text { мощностью } 500 \text { МВт }\left(n_{2}\right) \text { - тип №2; } \\
& \text { мощностью } 100 \text { МВт }\left(n_{3}\right) \text { - тип №3; } \\
& \text { мощностью } 10 \text { МВт }\left(n_{4}\right) \text { - тип №4. }
\end{aligned}
$$

Все ранги РУ имеют свои особенности, связанные с капиталовложениями, различными рисками и вероятным ущербом вследствие аварий. Поэтому условия многокритериальной оптимизации (МКО) состоят в нахождении соответствующего набора рангов РУ требуемой суммарной выходной мощности и с минимальными величинами рисков, ущерба и капитальных затрат.

Задача поиска оптимальных конфигураций системы РУ может быть представлена в виде оптимизационной задачи $F(\vec{n}) \rightarrow \min$, где $\vec{n}$ - вектор входных параметров, т. е. набора возможных РУ разных типов $\left(n_{1}, n_{2}, n_{3}, n_{4}\right)$, каждая из которых обладает своим набором функций рисков/ущерба/затрат.

Целевая функция $F(\vec{n})$ представляет собой в данном случае «вектор целей». Он строится как набор функций с учетом требований к оптимальности системы при условии их одинаковой важности (в данном случае - это максимальная выработка электроэнергии при минимально возможном уровне рисков/ущерба/затрат). Таким образом, имеет место задача многокритериальной оптимизации (МКО) $[5,6,11$ - 16].

$$
\begin{aligned}
& F(\vec{n}) \rightarrow \min \\
& \vec{n}=\left(n_{1}, n_{2}, n_{3}, n_{4}\right) \\
& n_{1,2,3,4} \in D \\
& 0 \leq n_{1} \leq N_{1}, \\
& 0 \leq n_{2} \leq N_{2}, \\
& 0 \leq n_{3} \leq N_{3}, \\
& 0 \leq n_{4} \leq N_{4},
\end{aligned}
$$


Многокритериальная оптимизация представляет собой минимизацию некоего вектора целей $\mathrm{F}(\mathrm{x})$, при этом могут быть наложены дополнительные ограничения или предельные значения:

Где $N_{1-4}$ максимально-допустимое количество РУ определенного типа.

Поскольку $F(\vec{n})$ - это вектор, любые его компоненты являются конкурирующими, поэтому единого решения поставленной задачи нет. Взамен этого для описания характеристик целей вводится концепция множества неулучшаемых решений оптимальность по Парето [5].

Оптимальность по Парето - это такое состояние некоторой системы, при котором значение каждого показателя (ущерб, риск, капитальные затраты), характеризующего систему, не может быть улучшено без ухудшения других.

Множество Парето представляет собой весь набор оптимальных решений (набора РУ) с минимальной функцией качества или функцией качества, удовлетворяющей определенным критериям с точки зрения критериев риска, ущерба, капитальных затрат.

Методики поиска неулучшаемых решений в многокритериальной оптимизации разнообразны. Среди наиболее используемых методов можно выделить стратегию взвешенных сумм, метод эпсилон-ограничений, метод достижения цели и др. Наиболее распространенный способ решения задачи многокритериальной оптимизации - сведение ее к задаче скалярной оптимизации, т. е. построение скалярной функции, минимум которой соответствует точке неулучшаемого решения исходной многокритериальной задачи.

При поиске оптимального набора рангов РУ при были выделены следующие критерии оптимальности:

наименьший ущерб $-F_{1}(\vec{n})$;

наибольшая производящая мощность набора РУ $-F_{2}(\vec{n})$;

наименьшие риски $-F_{3}(\vec{n})$;

наименьшие затраты $-F_{4}(\vec{n})$.

Как уже было сказано, $\vec{n}$ - набор РУ различных рангов мощности. Большинству анализируемых критериев оптимальности в дальнейшем будет назначена система оценок. За неимением опытного распределения этих величин будут назначены характерные показатели, определяющие тот или иной вес критерия по балльной системе, априори заданной на основе экспертных оценок.

Балльная оценка ущерба. Балльная система оценки ущерба соотносится с каждым из рангов РУ и характеризует ущерб в результате его аварии. Априори предполагается, что ущерб от аварии РУ большой мощности существенно выше, чем ущерб от аварии РУ меньшей мощности. Задается выражением $\mathrm{U}=\mathrm{x} * \mathrm{~K} * \mathrm{P}, \mathrm{x}-$ коэффициент, характеризующий тяжесть последствий - может быть выбран от 1 до 100 по усмотрению исследователя согласно литературным данным [5] по оценке ущерба от аварий на АC, К - капитальные затраты на блок определенной мощности; Р - вероятность запроектной аварии, расчетное значение вероятности берется согласно проектным данным вероятностного анализа безопасности или экспертно при их отсутствии.

Количественная оценка затрат. Закладывается, как совокупность всевозможных инвестиций для строительства АС. Учитывает стоимость оборудования, монтажа, строительства, изысканий, проектных и исследовательских работ, т.е. капитальная стоимость строительства. 
Предварительная экспертная оценка групп рисков. Балльная система оценки характеризует различные группы рисков/вызовов и показана в таблице 1 . В данный момент риски систематизированы по 6 группам - экономика, безопасность, воздействие на окружающую среду (ОС), риски от отработавшего ядерного топлива (ОЯТ) и радиоактивных отходов (РАО), инфраструктура, нераспространение ядерных материалов. Каждой из групп для оценки их взаимовлияния на общий показатель риска задан определенный вес.

Таблица 1. Балльная оценка рисков/вызовов для основных групп и показателей с интервалами неопределенности для четырех рангов мощности блоков АС

\begin{tabular}{|c|c|c|c|c|c|}
\hline Критерии & $\begin{array}{l}\text { Веса по } \\
\text { группам }\end{array}$ & АС БМ (B-1000) & $\begin{array}{l}\mathrm{AC} \mathrm{CM}(\mathrm{B}- \\
500)\end{array}$ & $\begin{array}{l}\text { AC MM } \\
(\mathrm{B}-100)\end{array}$ & $\begin{array}{l}\text { AC MM (B- } \\
10)\end{array}$ \\
\hline Экономика & \multirow{7}{*}{$0,1-0,7$} & 20 & 30 & 40 & 40 \\
\hline $\begin{array}{ll}\text { Капитальная } & \text { составляющая } \\
\text { стоимости э/э } & \end{array}$ & & $10-20$ & $40-60$ & $70-90$ & $70-90$ \\
\hline $\begin{array}{l}\text { Эксплуатационная } \\
\text { стоимости э/э }\end{array}$ & & $40-60$ & $30-50$ & $20-30$ & $10-20$ \\
\hline Найти инвестора & & $90-70$ & $40-60$ & $7-15$ & $5-10$ \\
\hline Срок строительства & & $90-80$ & $50-60$ & $5-10$ & $1-5$ \\
\hline Страхование имущественное & & $100-95$ & $40-60$ & $1-5$ & $1-2$ \\
\hline Экспортные возможности & & $70-50$ & $10-20$ & $1-5$ & $1-2$ \\
\hline Безопасность & \multirow{5}{*}{$0,2-0,7$} & 70 & $\mathbf{5 0}$ & 10 & 5 \\
\hline Пассивные системы & & $80-90$ & $40-50$ & $5-10$ & $1-5$ \\
\hline Последствия тяжелой аварии & & $70-90$ & $60-40$ & $5-10$ & $5-10$ \\
\hline $\begin{array}{l}\text { Останов по общей } \\
\text { (упущенная выгода) }\end{array}$ & & $70-80$ & $60-40$ & $1-5$ & $1-5$ \\
\hline Физическая безопасность & & $10-20$ & $40-60$ & $10-20-30$ & $10-20-30$ \\
\hline Воздействие на ОС & \multirow{3}{*}{$0,1-0,2$} & 70 & 40 & 5 & 1 \\
\hline Наличие площадок & & $70-80$ & $40-60$ & $1-5$ & $1-2$ \\
\hline Диверсификация СТВС & & $70-80$ & $20-30$ & $3-5$ & $1-3$ \\
\hline Радиационные от ОЯТ и РАО & \multirow{5}{*}{$0,2-0,3$} & 70 & 50 & 10 & 5 \\
\hline Обращение & & $80-90$ & $40-60$ & $10-20$ & $5-10$ \\
\hline Восприятие новых видов топлива & & $70-90$ & $40-60$ & $5-10$ & $5-10$ \\
\hline Вывод из эксплуатации & & $80-90$ & $40-60$ & $10-15$ & $10-15$ \\
\hline Приемлемость обществом & & $60-70$ & $40-60$ & $5-10$ & $5-10$ \\
\hline Инфраструктура & \multirow{6}{*}{$0,1-0,5$} & 20 & 40 & 10 & 5 \\
\hline Институциональная среда & & $10-20$ & $50-70$ & $80-90$ & $80-90$ \\
\hline Материально-техническая & & $40-50$ & $40-60$ & $1-5$ & $1-5$ \\
\hline Сетевая приемлемость & & $80-90$ & $30-40$ & $0-5$ & $0-1$ \\
\hline Неэлектрическое применение & & $90-80$ & $40-50$ & $1-5$ & $1-5$ \\
\hline Контур преобразования энергии & & 95 & 50 & $1-5$ & $1-5$ \\
\hline Нераспространение ЯМ & \multirow{2}{*}{$0,1-0,05$} & & & & \\
\hline Специфика топливного цикла & & $40-50$ & $40-50$ & $10-20$ & $10-20$ \\
\hline
\end{tabular}

Критерии оптимальности $F_{1-4}$ вычисляются по формулам:

$$
\begin{aligned}
& F_{1}=\min \left|\sum_{i=1}^{4} n_{i} d_{i}\right|, \\
& F_{2}=\min \left|P-\sum_{i=1}^{4} n_{i} p_{i}\right|, \\
& F_{3}=\min \left|\sum_{i=1}^{4} \sum_{j=1}^{6} n_{i} w_{j} r_{i j}\right|, \\
& F_{4}=\min \left|\sum_{i=1}^{4} n_{i} c_{i}\right|,
\end{aligned}
$$


Где $d_{i}$ - величина ущерба от определенного ранга РУ;

$p_{i}$ - величина мощности определенного ранга РУ;

$r_{i j}$ - показатель ј-ой группы риска для определенного ранга РУ;

$w_{j}$ - вес ј-ой группы риска;

$c_{i}$ - капитальные затраты на строительство определенного ранга РУ.

Общий процесс МКО может быть произведен для всех функций $F_{1-4}$ и записан следующим образом:

$$
\Phi=\min \left(\alpha_{1} F_{1}+\alpha_{2} F_{2}+\alpha_{3} F_{3}+\alpha_{4} F_{4}\right)
$$

Где $\alpha_{1-4}$ весовые коэффициенты, отражающие удельный вес величин той или иной функции. Их (весовые коэффициенты $\alpha_{1-4}$ ) предстоит задавать, чтобы обезразмерить сумму функций и исследовать отдельно влияние той или иной функции на общий процесс МКО:

$\alpha_{1}$ - весовой коэффициент для функционала ущербов - $F_{1}$;

$\alpha_{2}$ - весовой коэффициент для функции, отвечающей за суммарную мощность всего набора РУ $-F_{2}$;

$\alpha_{3}$ - весовой коэффициент для функции, отвечающей за интегральный риск для всего набора РУ $-F_{3}$;

$\alpha_{4}$ - весовой коэффициент для функции, отвечающей за инвестиционные затраты на строительство блока - $F_{4}$.

Даже в условиях небольшого диапазона изменения каждого из входных параметров $\left(n_{1}, n_{2}, n_{3}, n_{4}\right)$ число вариантов, которое нужно было бы просчитать для отыскания «самого оптимального» набора РУ простым перебором, равно $n_{1} * n_{2} * n_{3} * n_{4}$ и слишком велико.

В подобных условиях для ускорения поиска решения, когда необходимо выполнить численную оптимизацию функции в пространстве большой размерности, часто предпочтение отдается генетическому (или эволюционному) алгоритму.

По принципу действия ГА - это в первую очередь эволюционный алгоритм. Основная особенность алгоритма - скрещивание (комбинирование) полученных результатов. Сама идея алгоритма взята у природы. Путем перебора и отбора получается правильная и наиболее оптимальная «комбинация». Алгоритм (рис. 1) делится на три этапа: скрещивание, селекция (отбор) и формирование новой популяции. Если результат работы ГА не устраивает, эти этапы повторяются до тех пор, пока результат не начнет удовлетворять или произойдет одно из ниже перечисленных условий:

- количество поколений (циклов) достигнет заранее выбранного максимума;

- $\quad$ будет достигнуто минимальное значение функции качества $<\varepsilon$.

Далее следует более детально сказать об этапах работы ГА.

Создание начальной популяции. На этом шаге создается начальная популяция, которая, вполне возможно, окажется совсем не оптимальной, однако в дальнейшем алгоритм эту проблему должен исправить. Главное, чтобы популяция соответствовала заданному «формату» (соответствовала диапазону заданной конфигурации рангов РУ).

Скрещивание. Для этого используются 2 особи текущей популяции так, чтобы следующая особь могла унаследовать у родителей их черты. Для выбора родительских особей выбираются все особи из популяции. 
Мутация. Мутация схожа со скрещиванием. Для получения мутирующих особей выбирают некое количество особей из популяции и изменяют их в соответствии с заранее определенными критериями.

Селекция. Финальный этап. Выбираются из популяции (мутировавших и скрещенных особей) те, кто «пойдет дальше». При этом доля «выживших» после отбора определяется заранее и задается в виде параметра. Лишние особи отбрасываются.

Для решения задачи (3) при помощи генетического алгоритма была написана специальная программа алгоритм которой приведен на рис. 1.

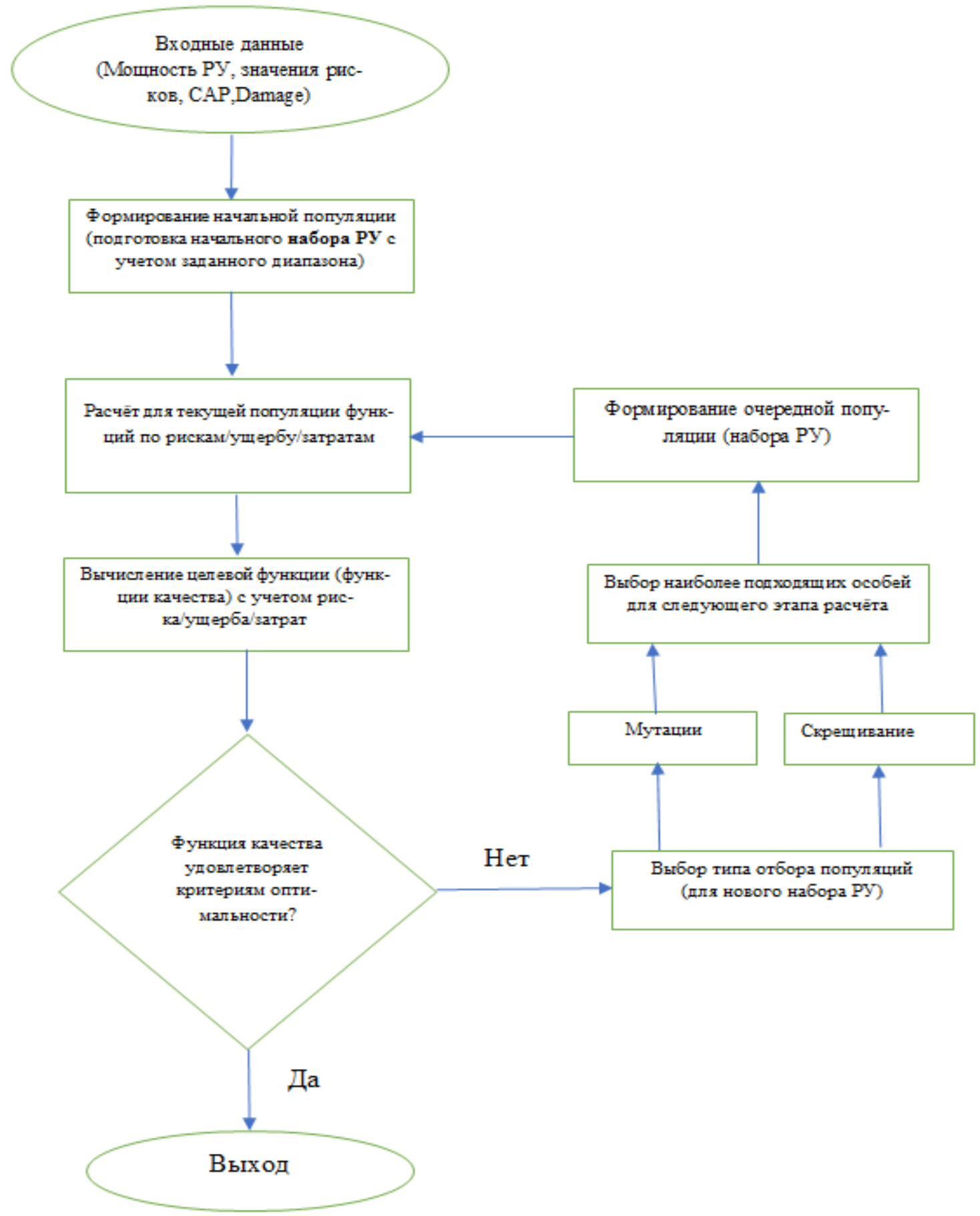

Рис. 1. Схема работы алгоритма МКО-ГА для поиска оптимальной конфигурации РУ 
Пример расчета оптимальной конфигурации системы РУ.

Приведенные далее численные результаты МКО иллюстрируют качественно-понятные и интуитивно ожидаемые результаты конфигурации мощностной инфраструктуры системы АЭ при сочетании означенных рисков и приоритетов.

В качестве примера рассматривается задача подбора оптимальной конфигурации мощностей РУ для системы АС с выработкой 15000 МВт электрической мощности. В качестве возможных вариантов рассматриваются 4 ранга РУ с мощностью 1000, 500, 100 и 10 МВт эл. Остальные входные параметры для расчёта приведены в таблице 2.

Таблица 2. Входные параметры для МКО определения набора РУ

\begin{tabular}{|c|c|c|c|c|c|c|}
\hline \multirow{2}{*}{\multicolumn{2}{|c|}{ Параметр }} & \multirow{3}{*}{$\begin{array}{l}\text { Вес групп, } \\
w_{j} \\
0,1-0,7 \\
\end{array}$} & \multicolumn{4}{|c|}{ Ранг РУ по мощности, рі , МВт } \\
\hline & & & \multirow{2}{*}{$\begin{array}{l}1000 \\
20 \\
\end{array}$} & \multirow{2}{*}{$\begin{array}{l}500 \\
30\end{array}$} & \multirow{2}{*}{$\begin{array}{l}100 \\
40 \\
\end{array}$} & \multirow{2}{*}{$\begin{array}{l}10 \\
50\end{array}$} \\
\hline 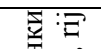 & Экономика & & & & & \\
\hline$\stackrel{\mathbb{E}}{E} \sum^{n}$ & Безопасность & $0,2-0,7$ & 70 & 50 & 10 & 1 \\
\hline 。沓 & $\begin{array}{lc}\begin{array}{l}\text { Воздействие } \\
\text { окружающую среду }\end{array} & \text { на } \\
\end{array}$ & $0,1-0,2$ & 70 & 40 & 5 & 1 \\
\hline 00 & ОЯТ и РАО & $0,2-0,3$ & 70 & 50 & 10 & 1 \\
\hline$\vec{\theta}$ & Инфраструктура & $0,1-0,5$ & 20 & 40 & 10 & 1 \\
\hline 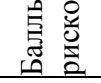 & $\begin{array}{l}\text { Нераспространение } \\
\text { ядерных материалов }\end{array}$ & $0,05-0,1$ & 50 & 50 & 5 & 2 \\
\hline \multicolumn{3}{|c|}{ Удельные капитальные затраты, у.е., $c_{i}$} & $5 \cdot 10^{3}$ & $10^{4}$ & $15 \cdot 10^{3}$ & $20 \cdot 10^{3}$ \\
\hline \multicolumn{3}{|c|}{ Ущерб×Вероятность, у.е., $d_{i}$} & $\begin{array}{l}2 \cdot 10^{11} \times 5 \cdot 10^{-7} \\
=10^{5}\end{array}$ & $\begin{array}{l}7 \cdot 10^{10} \times 5 \cdot 10^{-8} \\
=3,5 \cdot 10^{3}\end{array}$ & $\begin{array}{l}2 \cdot 10^{9} \times 5 \cdot 10^{-9} \\
=10\end{array}$ & 1 \\
\hline
\end{tabular}

Целевые приоритеты и предпочтения построения системы устанавливаются через назначение коэффициентов важности $\alpha_{1}, \alpha_{3}, \alpha_{4}$ соответствующих критериев безопасности, риска и экономичности (табл. 3). Для этих параметров задан диапазон изменений от 0 до 4-х, где 0 - отсутствие влияния данной величины, 4 - максимальное влияние. Для параметра $\alpha_{2}$ всегда задается максимальная величина 4, т.е. соблюдение требуемой мощности системы РУ (15 000MBт).

В качестве калибровочных рассмотрены два противоположных по смыслу варианта для иллюстрации качественно-понятных результатов - превалирование капитальных затрат (приоритет «минимальная стоимость», весовые коэффициенты $\alpha_{1-4}$, увеличивающие влияние той или иной функции качества, задаются следующим образом: $\alpha_{1}=4,\left(\alpha_{2}=4\right), \alpha_{3}=0$, $\alpha_{4}=0$ ); и превалирование ущерба (приоритет «максимальная безопасность», весовые коэффициенты: $\left.\alpha_{1}=0,\left(\alpha_{2}=4\right), \alpha_{3}=0, \alpha_{4}=4\right)$, результаты которых представлены в строках 1 и 2 табл. 3.

Вариант № 3 с превалированием совокупного риска в чистом виде нельзя считать калибровочным, так как этот параметр имеет много возможных вариаций с непредсказуемым заранее результатом и множество этих результатов явлются основным объектом исследования.

Приведенные в табл. 3 численные результаты оптимизации иллюстрируют интуитивно ожидаемую инфраструктуру АЭ при сочетании указанных рисков и приоритетов: в первом случае при приоритете экономичности строительства оптимальны РУ большой мощности с наименьшей удельной стоимостью; при включении влияния параметра ущерба (вариант № 2) оптимальны оказались РУ малой мощности. Результаты расчётов приведены для лучших (по сходимости расчетов) конфигураций системы АЭ с РУ разной мощности, что объясняет количественный разброс числа представленных решений (от 1 до 4 решений по вариантам).

Путем изменения весовых коэффициентов параметров можно рассмотреть мощностной ряд системы АС с разным уровнем приоритетов $\left(\alpha_{\mathrm{i}}=4\right)$ или предпочтений $\left(\alpha_{\mathrm{i}}=2\right.$ или 3$)$, выставляемых лицами принимающими решения, начиная от экстремальных, например, 
предпочтение экономичности перед другими критериями (в-т. 4), предпочтение безопасности (в-т. 5), или совокупному риску перед другими (в-т. 6), или когда все факторы равноважны (в-т. 7, 8), или наоборот - пренебрежимы (в-т. 9). Расчетный алгоритм также позволяет варьировать весомость разных групп рисков.

Таблица 3. Итоги расчёта мощностного спектра РУ $\left(n_{i}\right)$ для вариантов с приоритетами и преимуществами при заданной мощности системы 15 ГВт

\begin{tabular}{|c|c|c|c|c|c|c|c|}
\hline \multirow[b]{2}{*}{$\begin{array}{l}\text { Степень } \\
\text { важности } \\
\text { критериев }\end{array}$} & \multirow[b]{2}{*}{$\begin{array}{l}\text { № } \\
\text { вар. }\end{array}$} & \multirow[b]{2}{*}{ Критерии } & \multirow{2}{*}{$\begin{array}{l}\text { Коэф-ты } \\
\text { важности } \\
\text { ущерб/риск/зат } \\
\text { раты } \\
\alpha 1 / \alpha 3 \text { / } \alpha 4\end{array}$} & \multicolumn{4}{|c|}{ Ранг мощности блока, МВт } \\
\hline & & & & 1000 & 500 & 100 & 10 \\
\hline \multirow{4}{*}{ 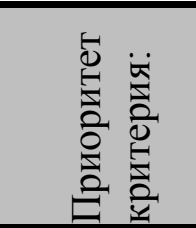 } & \multirow{2}{*}{1} & \multirow{2}{*}{ Экономика } & \multirow{2}{*}{$0 / 0 / 4$} & 14 & 2 & 0 & 0 \\
\hline & & & & 13 & 4 & 0 & 0 \\
\hline & 2 & Безопасность & $4 / 0 / 0$ & 0 & 0 & 137 & 130 \\
\hline & 3 & Риск & $0 / 4 / 0$ & 0 & 0 & 127 & 230 \\
\hline \multirow{5}{*}{ 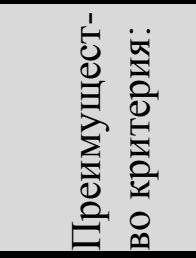 } & \multirow{3}{*}{4} & \multirow{3}{*}{ Экономика } & \multirow{3}{*}{$2 / 2 / 4$} & 0 & 30 & 0 & 0 \\
\hline & & & & 1 & 28 & 0 & 0 \\
\hline & & & & 2 & 26 & 0 & 0 \\
\hline & 5 & Безопасность & $4 / 2 / 2$ & 0 & 0 & 149 & 17 \\
\hline & 6 & Риск & $2 / 4 / 2$ & 0 & 2 & 140 & 0 \\
\hline \multirow{7}{*}{ 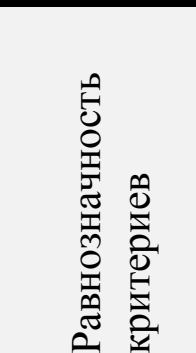 } & 7 & Равноважны & все $=4$ & 0 & 0 & 148 & 32 \\
\hline & \multirow{2}{*}{8} & \multirow{2}{*}{ Равнозначны } & \multirow{2}{*}{$\mathrm{Bce}=2$} & 0 & 1 & 143 & 20 \\
\hline & & & & 1 & 1 & 144 & 22 \\
\hline & \multirow{4}{*}{9} & \multirow{4}{*}{ Пренебрежимы } & \multirow{4}{*}{ все $=0$} & 6 & 0 & 85 & 50 \\
\hline & & & & 8 & 8 & 7 & 230 \\
\hline & & & & 2 & 5 & 98 & 70 \\
\hline & & & & 7 & 11 & 20 & 50 \\
\hline
\end{tabular}

Примечание: $\alpha 2=4$ для всех вариантов.

Для некоторых вариантов приведены несколько близких к оптимуму решений.

Заключение. Применение методов математического моделирования для поддержки принятия решений на разных уровнях управления энергетическим комплексом страны имеет большой научный и практический потенциал. При этом нахождение и применение новых подходов к оптимизации обогащает этот опыт и открывает новые сферы применения. Рассмотренный в статье генетический алгоритм применительно к проблеме нахождения оптимального спектра мощностей атомных реакторов с учетом глобальных рисков, затрат и выгод показал, что возможно добиваться снижения рисков управленческими решениями при использовании многокритериального подхода при разработке стратегий. В условиях смены концепций и переосмысления энергетического уклада появляется аргументированная основа перехода к локальной и распределенной энергогенерации, как эффективного пути снижения потерь в тепло- и электро-сетях; упрощения инфраструктурных проблем.

Помощь результатов оптимизационных исследований для смещения интереса проектировщиков в сторону использования блоков средней и малой мощности позволит создать своего рода «превентивную страховку» от возникновения тяжелых ядерных аварий с крупными ущербами экономике, здоровью, окружающей среде (в отличие от традиционной страховки после инцидента). Также это направление атомной энергетики позволит расширить зоны применимости АЭ в региональной энергетике и, соответственно, рыночные 
ниши. Но всё же основная преследуемая цель такой структурной переориентации - снижение совокупного риска развивающейся широкомасштабной Системы АЭ.

Благодарности. Работа выполнена при поддержке гранта НИЦ «Курчатовский институт» (№ 2220 от 23.10.2020)

\section{СПИСОК ЛИТЕРАТУРЫ}

1. Locatelli, G. and Mancini, M. The role of the reactor size for an investment in the nuclear sector: an evaluation of not-financial parameters./ ELSEVIER Ltd. Progress in Nuclear Energy. 2011. 53 (2). Pp. 212-222

2. Energy source for human demand. Sadao Hattori /in Advanced Nuclear Systems Consuming Excess Plutonium/ Kluwer Academic Publishers, Netherlands. 1997. Pp. 69 - 77.

3. Домашняя страница оптимизационного модуля PEST. Режим доступа: https://pesthomepage.org/software (дата обращения 20.04.2021).

4. Домашняя страница оптимизационного модуля Ostrich. Режим доступа: https://www.civil.uwaterloo.ca/envmodelling/Ostrich.html (дата обращения 20.04.2021).

5. Кононюк А.Е. Дискретно-непрерывная математика. Учебное пос. Кн. 5. Ч. 3. Киев: Освіта України. 2012. 520 с.

6. Е. В. Волкова, И. А. Расторгуев, А. В. Расторгуев. Численное моделирование для обоснования системы инженерной защиты г. Казани. Водоснабжение и санитарная техника. 2010. №12.

7. Саркисов А.А. Феномен восприятия общественным сознанием опасности, связанной с ядерной энергетикой //Научно-технические ведомости СПбГПУ. 2012 г. №3-2(154). C. 9-21.

8. Догов А.А. Разработка и применение алгоритмов, методов и средств компоновки технологического оборудования и организации помещений АЭС - АО ИК «АСЭ» (АО «НИАЭП»). Режим доступа: $\quad$ http://www.innov-rosatom.ru/vertical/niaep 189c97b30fb4919d2317397df639bccab.pdf (дата обращения 20.04.2021).

9. Ю.Б. Воробьев, П. Кудинов, М. Ельцов, К. Кёоп, К.Н. Чыонг Ван. Применение информационных технологий (генетические алгоритмы, нейронные сети, параллельные вычисления) в анализе безопасности АЭС // Труды Института системного программирования РАН, .c. 137 - 156. Режим доступа: https://cyberleninka.ru/article/v/primenenie-informatsionnyh-tehnologiy-geneticheskiealgoritmy-neyronnye-seti-parallelnye-vychisleniya-v-analize-bezopasnosti-aes обращения 20.04.2021).

10. Емельянов В. В., Курейчик В. В., Курейчик В. М. Теория и практика эволюционного моделирования. М.: Физматлит. 2003.

11. Баланин А.Л., Гроль А.В., Гурин А.В., Крылов Д.А., Невиница В.А., Теплов П.С., Фомиченко П.А., Марова Е.В., Маслов А.М., Шепелев С.Ф. Развитие многокритериальных подходов к оценке конкурентоспособности электростанций с атомным и неатомным энергоисточником // Энергетические установки и технологии. 2018. 4(4). С. 67-82.

12. Б.И. Яцало, С.В. Грицюк, В.И. Диденко. Система многокритериального анализа решений DecernsMCDA и ее практическое применение // Программные продукты и системы. 2014г. № 2 .

13. В.А. Шакиров, П.С. Панкратьев. Методика многокритериального двухуровневого анализа пунктов размещения электростанций //Искусственный интеллект и принятие решений. 1/2017. 
14. Кравченко Т.К., Авдеев Ю.В. Развитие систем поддержки принятия решений с использованием метода PROMETHEЕ // Актуальные проблемы гуманитарных и естественных наук. 2010. № 9. С. 67-70.

15. Е.А. Елтаренко. Оценка и выбор решений по многим критериям. Учебное пособие. Москва. МИФИ. 1995. 111с.

16. Methodology for the assessment of innovative nuclear reactors and fuel cycles. Report of Phase 1B (first part) of the International Project on Innovative Nuclear Reactors and Fuel Cycles (INPRO). Vienna: IAEA. 2004. IAEA-TECDOC-1434.

UDK 004.02, 621.039

\title{
METHODOLOGY OF SEARCHING FOR THE OPTIMAL CONFIGURATION OF A NUCLEAR PLANT SYSTEM TAKING INTO ACCOUNT THE INFLUENCE OF RISKS, CAPITAL COSTS AND DAMAGE FROM ACCIDENTS
}

\author{
Ivan A. Rastorguev
}

Ph.D., senior researcher, e-mail: rastorguev_ia@ nrcki.ru

Tatiana D. Shchepetina

Ph.D., head of laboratory "Perspective Concepts" e-mail: Schepetina_TD@ nrcki.ru

National Research Center "Kurchatov Institute"

123182, Moscow, sq. Kurchatova, 1

\begin{abstract}
Annotation. The problem of finding the optimal power spectrum of nuclear reactors for the projecting power system for the whole country is presented. It considers a large contribution of NPP and formalized in the form of a multicriteria optimization problem. Potential damage reduction from various risk factors and construction costs at maximum power generation is estimated. The genetic algorithm is proposed as solver algorithm. Recommendations are given for optimizing the power range of reactor plants on the basis of the performed calculations and reduce the overall risks of NPP operation during strategic planning.
\end{abstract}

Keywords: nuclear power, reactor plants, power capacity of a unit, safety, damage, risk, capital investments, damage minimization, evolutionary algorithm, optimization.

Acknowledgments. This work was supported by a grant from the National Research Center "Kurchatov Institute" (No. 2220 dated 23.10.2020)

\section{REFERENCES}

1. Locatelli, G. and Mancini, M. The role of the reactor size for an investment in the nuclear sector: an evaluation of non-financial parameters./ ELSEVIER Ltd. Progress in Nuclear Energy. 2011. 53 (2). Pp. 212-222

2. Energy source for human demand. Sadao Hattori /in Advanced Nuclear Systems Consuming Excess Plutonium/ Kluwer Academic Publishers, Netherlands. 1997. Pp. 69 - 77.

3. Homepage of PEST optimization module. Available at: https://pesthomepage.org/software (accessed 20.04.2021).

4. Homepage of optimization module Ostrich. Available at: https://www.civil.uwaterloo.ca/envmodelling/Ostrich.html (accessed 20.04.2021).

5. Kononyuk A.E. Diskretno-nepreryvnaya matematika. Uchebnoe pos. Kn. 5. CH. 3. [Discrete-continuous mathematics. Educational encyclopedia. Book 5. Part 3.] Kiev: Osvita Ukrainy. 2012. 520 p.

6. E. B. Volkova, I. A. Rastorguev, A. V. Rastorguev. Chislennoe modelirovanie dlya obosnovaniya sistemy inzhenernoj zashchity g. Kazani. [Numerical modeling for 
justification of engineering protection system of Kazan]. Vodosnabzhenie i sanitarnaya tekhnika $=$ Water supply and sanitary engineering. 2010. № 12 .

7. Sarkisov A.A. Fenomen vospriyatiya obshchestvennym soznaniem opasnosti, svyazannoj s yadernoj ener-getikoj [Phenomenon of Public Perception of Danger Associated with Nuclear Power Engineering] // Nauchno-tekhnicheskie vedomosti SPbGPU = Scientific and Technical Bulletin of Saint Petersburg State Polytechnic University. 2012 г. №3-2(154). Pp. 9-21.

8. A.A. Dogov. Razrabotka i primenenie algoritmov, metodov i sredstv komponovki tekhnologiche-skogo oborudovaniya i organizacii pomeshchenij AES - AO IK «ASE» (AO «NIAEP»). [Development and Application of Algorithms, Methods and Means of Layout of Technological Equipment and Organization of NPP Premises - JSC NIAEP]. Available at: http://www.innov-rosatom.ru/vertical/niaep /89c97b30fb4919d2317397df639bccab.pdf (accessed 20.04.2021).

9. Y.B. Vorobyov, P. Kudinov, M. Eltsov, K. Koop, K.N. Truong Van. Primenenie informacionnyh tekhnologij (geneticheskie algoritmy, nejronnye se-ti, parallel'nye vychisleniya) v analize bezopasnosti AES. [The Application of Information Technologies (Genetic Algorithms, Neural Networks, Parallel Computing) in Analysis of NPP Safety] // Trudy Instituta sistemnogo programmirovaniya RAN = Proc. Pp.137 - 156. Available at: https://cyberleninka.ru/article/v/primenenie-informatsionnyh-tehnologiy-geneticheskiealgoritmy-neyronnye-seti-parallelnye-vychisleniya-v-analize-bezopasnosti-aes (accessed 20.04.2021).

10. Emelyanov V.V., Kureichik V.V., Kureichik V.M. Teoriya i praktika evolyucionnogo modelirovaniya [Theory and practice of evolutional modeling]. Moscow: Fizmatlit. 2003.

11. Balanin A.L., Grol A.V., Gurin A.V., Krylov D.A., Nevinitsa V.A., Teplov P.S., Fomichenko P.A., Marova E.V., Maslov A.M., Shepelev S.F. Razvitie mnogokriterial'nyh podhodov $\mathrm{k}$ ocenke konkurentosposobnosti elektro-stancij $\mathrm{s}$ atomnym i neatomnym energoistochnikom [Development of multi-criteria approaches to assessing the competitiveness of power plants with nuclear and non-nuclear power sources] // Energeticheskie ustanovki i tekhnologii $=$ Power Plants and Technologies. 2018. 4(4). Pp. 67-82.

12. B.I. Yatsalo, S.V. Gritsyuk, V.I. Didenko. Sistema mnogokriterial'nogo analiza reshenij DecernsMCDA i ee prakticheskoe primenenie [DecernsMCDA multicriteria solution analysis system and its practical application] // Programmnye produkty i sistemy = Software products and systems. 2014. № 2.

13. V.A. Shakirov, P.S. Pankratiev. Metodika mnogokriterial'nogo dvuhurovnevogo analiza punktov razmeshcheniya elek-trostancij [Methodology of multi-criteria two-level analysis of power plant locations] // Iskusstvennyj intellekt i prinyatie reshenij = Artificial Intelligence and Decision Making. 1/2017.

14. Kravchenko T.K., Avdeev Yu.V. Razvitie sistem podderzhki prinyatiya reshenij s ispol'zovaniem metoda PROMETHEE [Development of decision support systems using PROMETHEE method] // Aktual'nye problemy gumanitarnyh i estestvennyh nauk = Actual problems of the humanities and the natural sciences. 2010. № 9. Pp. 67-70.

15. E.A. Eltarenko. Ocenka i vybor reshenij po mnogim kriteriyam. Uchebnoe posobie [Evaluation and choice of decisions by multiple criteria. Tutorial]. Moscow. MEPHI. 1995. $111 \mathrm{p}$.

16. Methodology for the assessment of innovative nuclear reactors and fuel cycles. Report of Phase 1B (first part) of the International Project on Innovative Nuclear Reactors and Fuel Cycles (INPRO). Vienna: IAEA. 2004. IAEA-TECDOC-1434. 